\title{
Differential Cytokine Release from Brain Microvascular Endothelial Cells Treated with Dexamethasone and Multiple Sclerosis Patient Sera
}

\author{
Malgorzata Burek $^{1^{*}}$, Aiden Haghikia ${ }^{2}$, Ralf Gold ${ }^{2}$, Norbert Roewer ${ }^{1}$, Andrew Chan ${ }^{2}$ and Carola Y Förster ${ }^{1}$ \\ ${ }^{1}$ University of Wurzburg, Department of Anaesthesia and Critical Care, Wurzburg, Germany \\ ${ }^{2}$ Ruhr University Bochum, Department of Neurology, St. Josef-Hospital, Bochum, Germany
}

"Corresponding author: University of Würzburg, Department of Anaesthesia and Critical care, Oberdüerrbacher str. 6, 97080 Würzburg, Germany, Tel: +4993120130046; Fax: +4993120130019; E-mail: Burek_M@ukw.de

Received date: February 20, 2014, Accepted date: April 22, 2014, Published date: April 29, 2014

Copyright: $\odot 2014$ Burek M, et al. This is an open-access article distributed under the terms of the Creative Commons Attribution License, which permits unrestricted use, distribution, and reproduction in any medium, provided the original author and source are credited.

\begin{abstract}
Objective: Multiple sclerosis (MS) is a neurodegenerative disorder of the central nervous system (CNS). Damage of the blood-brain barrier integrity is a key pathogenic event leading to the migration of lymphocytes into the CNS and subsequent demyelination. This process is tightly regulated by chemokines and cytokines which are target of therapeutic strategies in MS, such as anti-inflammatory glucocorticosteroid treatment. Here, we examine the effects of dexamethasone-treatment and MS patient sera on the expression of cytokines and chemokines in brain microvascular cell line, cEND in vitro.
\end{abstract}

Methods: We conducted 96-well Mouse Cytokines and Receptors qPCR arrays to quantitatively compare the cytokine and chemokine expression profiles after treatment with dexamethasone. For selected cytokines, we studied the effects of pre-treatment with MS patient sera from active phase of disease (exacerbation) or in relapse (remission) in combination with dexamethasone.

Results: After dexamethasone treatment, colony stimulating factor 3 (Csf3) and interleukin 17F (IL17f) were significantly up-regulated, whereas the chemokine (C-C motif) ligand 12 (CCL12/MCP-5), chemokine (C-X-C motif) receptor 3 (CXCR3) and kit oncogene (Kit) were significantly down-regulated. These results were confirmed in qRTPCR using gene-specific primers. For Csf3 and CCL12 we analyzed the dexamethasone-mediated changes in protein levels secreted into the cell culture medium. Dexamethasone treatment increased the release of Csf3 into the culture medium and decreased the release of CCL12 by cEND. Additionally, we examined the effects of MSpatient sera on dexamethasone-induced cytokine secretion. Pretreatment with MS-patient serum from exacerbation phase augmented dexamethasone effects on Csf3 and CCL12 release in CEND cells. The expression of Csf3- and CCL12-receptors was demonstrated on protein and mRNA level in CEND cells.

Conclusion: We identified Csf3 (G-Csf) and CCL12 as cytokines differentially regulated by dexamethasone on mRNA and protein level. This effect was even more pronounced after pretreatment with MS patient serum, especially from patients with acute relapses.

Keywords: Multiple sclerosis; Blood-brain barrier; Glucocorticoids; Dexamethasone; Cytokine; Chemokine; Patient sera; Csf3; CCL12

\section{Introduction}

Multiple sclerosis (MS) is a neuroinflammatory disorder characterized by the infiltration of pathogenic T cells into the CNS, which react with myelin antigens [1]. The majority of MS patients suffer initially from the relapsing-remitting form of the disease, which is characterized by attacks of neurological dysfunction followed by a gradual improvement. The exacerbations in relapsing-remitting MS are initiated by breakdown of the blood-brain barrier (BBB), which causes infiltration of activated immune effector cells into the brain and subsequent tissue damage [2,3]. Disruption of the BBB integrity is partially caused by Matrix metalloproteinases (MMPs) which are activated by proinflammatory cytokines increased during exacerbations of MS [4,5]. The levels of MMP-9, MMP-2 and tight junction protein claudin-5 and occludin change in different stages of MS [6]. High-dose glucocorticosteroid (GC) "pulse"-therapy is approved as first line therapy of relapses in MS [7]. The therapeutic GC-mediated effects on peripheral T cells in MS are widely studied $[8,9]$. In endothelial cells of the BBB, GC treatment reduces MMP levels and activity thereby stabilizing the BBB through higher expression of tight and adherens junction proteins [10-12].

Cytokines and chemokines play an important role in CNS neuroinflammatory disorders. The chemokine family is divided into four subfamilies: $\mathrm{CC}, \mathrm{CXC}, \mathrm{CX}_{3} \mathrm{C}$ and $\mathrm{XC}$, based upon the position of the first and second conserved cysteine $(\mathrm{C})$ residues within the mature peptides. Chemokines receptors are named as $\mathrm{G}$ protein-coupled receptors for $\mathrm{CC}, \mathrm{CXC}, \mathrm{CX}_{3} \mathrm{C}$ and $\mathrm{XC}$ chemokines [13]. Human primary brain endothelial cells as well as the human brain microvascular endothelial cell line hCMEC/D3 showed the constitutive expression of CXCL8 and CXCL2. CXCL10 and CCL5 were undetectable in resting endothelial cells but were secreted in response to proinflammatory cytokines, TNFa and IFN- $\gamma$ [14]. Exposure of endothelial cell monolayers to dexamethasone is known to increase the expression of tight junction proteins, therefore substantially decreasing endothelial cell permeability [15]. 
Previously, we demonstrated barrier compromising effects of MS patient sera using an in vitro model of the BBB, a mouse brain microvascular endothelial cell line cEND $[10,16]$. In this study we further characterized cEND and analyzed their cytokine and chemokine expression in response to dexamethasone or MS patient sera treatment in vitro. We identified Csf3 and CCL12 genes as strong responders to dexamethasone treatment in brain vascular endothelium in vitro.

\section{Methods}

\section{Reagents}

Dexamethasone was purchased from Sigma. Stock solution was prepared in ethanol and kept at $-20^{\circ} \mathrm{C}$. Desired dilution was made directly before the experiment in cell culture medium. The final concentration of ethanol in the treatment medium was lower than $0.01 \%(\mathrm{v} / \mathrm{v})$. Dexamethasone was used at final concentration of 100 $\mathrm{nM}$, which has been chosen according to the previous kinetics studies performed on brain ECs [10]. Control cells were treated with diluted vehicle only.

\section{Patients and patient sera}

The study was approved by local ethics committees. MS patient sera were prepared and used in cell culture as previously described [16]. In brief, sera of RR-MS patients with different time points of last GC treatment and three normal control donors, devoid of clinical signs of infections or immunotherapies, were chosen for the following experimental set-ups. Blood was obtained by venipuncture using CPT Vacutainer containing Na-citrate as anticoagulant (Becton Dickinson). Vacutainers were centrifuged for $20 \mathrm{~min}$ at $1650 \mathrm{~g}$. Serum with lymphocytes was transferred to new Falcon tubes, centrifuged again at $1200 \mathrm{rpm}$ for $10 \mathrm{~min}$ and serum supernatant was removed and stored in aliquots until use. For the cell culture, pooled serum samples from healthy controls and MS patients were used.

\section{Endothelial cell culture}

Cerebral microvascular ECs were isolated as described [10,17]. Cells were cultivated in Dulbecco's Modified Eagle's Medium (DMEM) (Sigma) supplemented with $10 \%$ fetal calf serum (FCS) and $100 \mathrm{IU} / \mathrm{ml}$ penicillin/streptomycin on collagen I coated flasks. Cells were maintained in $37^{\circ} \mathrm{C}$ and $5 \% \mathrm{CO}$. Cells were grown to confluence and transferred to serum-low medium ( $2 \%$ FCS) for differentiation.

\section{Mouse cytokines and receptors 96 stellARray ${ }^{\mathrm{m}}$ qPCR array}

Mouse Cytokines and Receptors 96 StellARray ${ }^{\text {me }}$ qPCR arrays (Lonza) ( $\mathrm{n}=3$ per condition) were conducted to compare the cytokine expression in cEND treated for 24 hours with vehicle only or $100 \mathrm{nM}$ dexamethasone. The qPCR analyses were performed according to manufacturer instructions using the $\mathrm{SYBR}^{\circledR}$ Green Master Mix (Life Technologies) and ABI PRISM 7300 system (Life Technologies). The gene expression fold changes and statistical tests were estimated by Global Pattern Recognition ${ }^{\text {TM }}$ (GPR) Data Analysis Tool (Lonza).

\section{Real-time PCR}

For real-time PCR, RNA was isolated from cells treated or untreated with dexamethasone using NucleoSpin ${ }^{\circ}$ RNA II Kit (Macherey-Nagel). One microgram of RNA was used for cDNA synthesis with High Capacity cDNA Reverse Transcription Kit (Life Technologies). Real-time PCR was performed using the SYBR Green Master Mix (Life Technologies). Primers were designed using Primer3 Software and synthesized by Eurofins MWG Operon and tested for PCR efficiency. The primer sequences were as follows: CXCL2: aagtttgccttgaccctgaa (sense), aggcacatcaggtacgatcc (anti-sense); CXCL12: agtagtggctccccaggttt (sense), gagacagtcttgcggacaca (antisense); CCL12: gggaagctgtgatcttcagg (sense), gggaacttcagggggaaata (anti-sense); Il2rb: atgacccctgtgtggaagag (sense), ggatgctagggagggaagtc (anti-sense); Ill7f: gtgttcccaatgcctcactt (sense), gtgcttcttccttgccagtc (anti-sense); CCL7: tgaaaaccccaactccaaag (sense), cattccttaggcgtgaccat (anti-sense); Kit: ttatcctttaggccgtgtgg (sense), tgtggccccttaagtacctg (anti-sense); Il23a: gactcagccaactcctccag (sense), gcactaagggctcagtcag (anti-sense); Csf3: ctcaactttctgcccagagg (sense), taggtggcacacaactgctc (anti-sense); Csf3-R: gagctgtggacacatcgaga (sense), aggaaggcctgggtgtagtt (anti-sense); Ccr2: agagagctgcagcaaaaagg (sense), ggaaagaggcagttgcaaag (anti-sense); GAPDH: ttcaccatggagaaggc (sense), ggcatcgactgtggtcatga (anti-sense). All PCR reactions were performed in triplicate for each target. Data were acquired with the ABI PRISM 7300 system (Life Technologies). The ABI PRISM 7300 SDS software (relative quantification study) was used to determine the threshold cycle $\left(C_{\mathrm{t}}\right)$ for each reaction and gene expression was normalized to the expression of the endogenous housekeeping gene GAPDH based on the $2^{-} \Delta \Delta \mathrm{Ct}$ method.

\section{Western blot analysis}

cEND cells were grown to confluence, differentiated and treated with dexamethasone or vehicle only in serum-free medium for 24 hours. For Western blot analyses, cells were lysed in RIPA buffer supplemented with proteases inhibitors cocktail (Roche). Protein contents were quantified by BCA protein Assay Kit (Thermo Fisher Scientific) and $40 \mu \mathrm{g}$ of protein were loaded on SDS-polyacrylamide gels for Western blot analysis. For immunoblotting, proteins were transferred to Hybond nitrocellulose membranes (Promega) which were blocked with $10 \%(\mathrm{w} / \mathrm{v})$ low fat milk in PBS and incubated overnight at $4^{\circ} \mathrm{C}$ with the respective primary antibody in blocking soultion. Rabbit polyclonal antibody against mouse Csf3R (1:200, \#sc-694, Santa Cruz Biotechnology) and goat polyclonal anti-mouse Ccr2 (1:1000, \#ab25788, Abcam) and mouse monoclonal anti- $\beta$-actin antibody (Sigma; 1:5000) were used. Respective horseradishperoxidase-labelled secondary antibodies (GE Healthcare) diluted 1:3000 were used. For detection, Enhanced Chemiluminescence (ECL) detection kit (Promega) and FluorChem FC2 Multi-imager II (Alpha Innotech) with CD camera were used. Intensity of protein bands was calculated with the software Alpha View (Alpha Innotech).

\section{Detection of secreted proteins}

cEND cells were grown to confluence, differentiated and treated with dexamethasone or vehicle only in serum-free medium for 24 hours. For pretreatment with MS-patient sera, the cells were incubated 24 hours with $5 \%$ patient sera. Then the cells were washed with phosphate buffer saline (PBS) (PAA Laboratories) and treated for additional 24 hours with $100 \mathrm{nM}$ dexamethasone in serum free medium. The same amounts of cell culture medium were collected, mixed with ice cold acetone in proportion 1 to 6 , followed by 2 hours incubation at $-80^{\circ} \mathrm{C}$. Precipitated proteins were centrifuged at $13000 \mathrm{~g}$ for $10 \mathrm{~min}$ at $4^{\circ} \mathrm{C}$. The resulting pellets were air dried and resuspended in 1.5x Tris-Tricine sample buffer (Tris-HCL pH 6.8, 50\% glycerol, $10 \%$ SDS, 10 mg Coomassie Blue G-250). The samples were loaded on 
Page 3 of 8

16.5\% Tris-Tricine Gel (BioRad). For Western blot detection of secreted G-CSF and CCL12 rabbit anti-mouse G-Csf antibody (Lifespan Biosciences \#LS-C42075/28157, at 1:500 dilution) and rabbit anti-mouse CCL12 (AbD Serotec \#AAM45, at 1:500 dilution) antibodies were used. As secondary antibody the horseradishperoxidase-labelled donkey anit-rabbit IgGs (GE Healthcare) diluted 1:3000 were used. For detection, Enhanced Chemiluminescence (ECL) detection kit (Promega) and FluorChem FC2 Multi-imager II (Alpha Innotech) with $\mathrm{CD}$ camera were used. Optical density values of protein bands were calculated with the software Alpha View (Alpha Innotech). As a loading control, Coomassie stained gels loaded with the same amount of medium-protein sample were used.

\section{Statistical analysis}

All data are presented as mean \pm standard deviation (SD) from at least three independent experiments. Statistical analysis was performed with SigmaStat 3.1 Software. For statistical comparison of two groups, we used an unpaired 2-tailed Students t-test; for the comparison of multiple groups, we used 2-way ANOVA followed by Tukey post hoc test. Differences were considered significant for $p$ values less than 0.05 .

\section{Results}

\section{Dexamethasone-mediated changes in the expression of cytokines mRNA in cEND}

There are several reports in the literature describing the expression of selected cytokines in brain microvascular endothelial cells (ECs)
$[14,18,19]$. We have previously demonstrated that brain microvascular endothelial cell line cEND responds to TNFa treatment in vitro $[20,21]$. However, the cEND cells were not yet characterized in terms of expression of cytokines and their receptors. We thus amplified mRNA of different cytokines present in differentiated untreated cEND and then analysed the dexamethasone-mediated changes of cytokine expression in these cells. We performed 96-well real-time transcription polymerase chain reaction (qRT-PCR) arrays (Table 1). The obtained results showed significant dexamethasone-mediated changes in the expression of chemokines and cytokines involved in the inflammation and immune response: Csf3 (10.4-fold), CCL12 (-6.7-fold), CXCR3 (-4.2-fold), Il17f (5.3-fold) and Kit (-3.6-fold). The results obtained with StellArray PCR array were reproduced in the qPCR using gene specific primers which confirmed upregulation of respective genes (Csf3, Il17f, CXCL2, CXCL12, Il2rb, Il23a) (Figure 1). Similarly, the three down-regulated genes (CCL7, CCL12 and Kit) were also found to be down-regulated by real time PCR analysis. Levels of expression changes due to dexamethasone treatment were variable, but Csf3 showed the highest-fold up-regulation among gene of the six selected genes by PCR-array analysis, and the CCL12 showed the highest-fold down-regulation. In conclusion, both methods (commercially available qPCR arrays and quantitative real-time PCR) demonstrated similar dexamethasone-mediated changes in the expression of cytokines by cEND. Detection of mRNA for cytokines and chemokines as shown in Table 1 and Figure1 suggests local production as well as the ability of brain microvascular endothelial cells to respond in situ to inflammatory stimuli.

\begin{tabular}{|l|l|l|l|l|}
\hline Gene Symbol & GenBank Accession Number & Fold change & P Value & Gene Name \\
\hline Csf3 & NC_000077 & 10.3 & 0.01327 & colony stimulating factor 3 (granulocyte) \\
\hline CCL12 & NM_011331 & -6.7 & 0.03012 & chemokine (C-C motif) ligand 12 \\
\hline CXCR3 & NM_009910 & -4.2 & 0.03602 & chemokine (C-X-C motif) receptor 3 \\
\hline II17f & NM_145856 & 5.3 & 0.03828 & interleukin 17F \\
\hline Kit & NM_001122733 & -3.6 & 0.04448 & kit oncogene \\
\hline CXCL2 & NM_009140 & 9.4 & 0.05083 & chemokine (C-X-C motif) ligand 2 \\
\hline Ccr5 & NM_009917 & -5.5 & 0.07481 & chemokine (C-C motif) receptor 5 \\
\hline CXCL12 & NM_001012477 & 2.7 & 0.08845 & chemokine (C-X-C motif) ligand 12 \\
\hline II22ra1 & NM_178257 & 4.8 & 0.09177 & interleukin 22 receptor, alpha 1 \\
\hline II2rb & NM_008368 & 2.8 & 0.09458 & interleukin 2 receptor, beta chain (II2rb) \\
\hline CCL7 & NM_013654 & -2.5 & 0.09807 & chemokine (C-C motif) ligand 7 \\
\hline II23a & NM_031252 & 2.9 & 0.10258 & interleukin 23, alpha subunit p19 \\
\hline
\end{tabular}

Table 1: Quantitative dexamethasone-mediated changes in cEND mRNA expression obtained with Mouse Cytokines and Receptors 96 StellARray ${ }^{\mathrm{m}} \mathrm{qPCR}$ array.

cEND were left untreated or were treated with $100 \mathrm{nM}$ dexamethasone for 24 hours. Mouse Cytokines and Receptors 96 StellARray ${ }^{\text {"m }}$ qPCR arrays were conducted according to manufacturers' instruction ( $n=3$ per treatment). The gene expression fold changes and p values were calculated by Global Pattern Recognition ${ }^{\mathrm{TM}}$ (GPR) Data Analysis Tool (Lonza). 


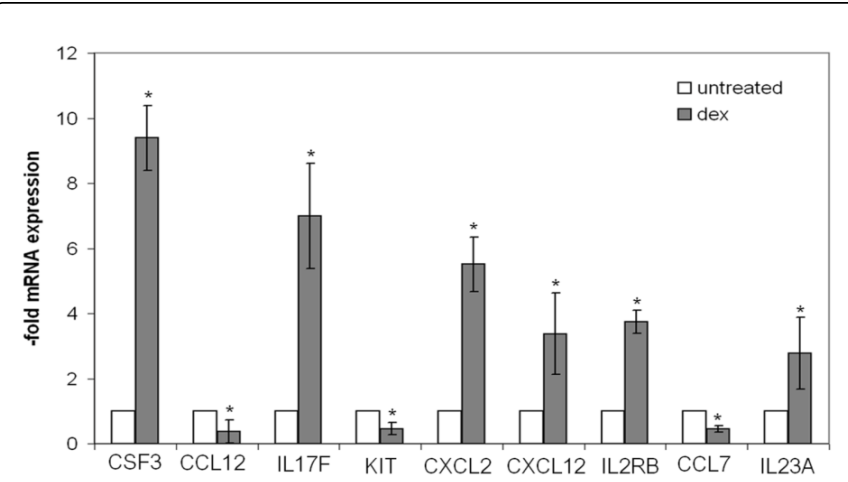

Figure 1: Dexamethasone-mediated changes in mRNA expression of selected cytokines in cEND cells

Confluent and differentiated cEND cells were treated with $100 \mathrm{nM}$ dexamethasone (dex) for 24 hours followed by total RNA extraction and quantitative real-time PCR with gene specific primers described in Methods section. Cytokine expression was normalized to GAPDH and expressed as fold over untreated cells, which was set as 1 . The data (mean $\pm \mathrm{SD}$ ) present the results of three independent experiments. Statistical analysis was performed by $\mathrm{t}$-test; ${ }^{*} \mathrm{p}<0.05$.

\section{Differential effects of dexamethasone treatment on G-Csf and CCL12 secretion}

To study the influence of dexamethasone treatment on the protein level, we selected Csf3 which was significantly increased on mRNA level. Granulocyte-colony stimulating factor, G-Csf (other names CSF3OS, C17orf33), a key hemopoietic factor of the myeloid lineage is encoded by Csf3 gene [22]. As a second protein, we selected CCL12 (other names MCP-5 and Scya12) [23], whose mRNA was significantly decreased after dexamethasone treatment. Since the cytokines and growth factors are rapidly released from the cells, we used the cell culture medium from cells cultured without serum for Western blot analysis.

While basal G-Csf release in untreated cells was hardly detectable, cells treated with dexamethasone released 2.5 - fold $(p=0.029)$ higher amounts of G-Csf into the medium (Figure 2A). CCL12 was highly secreted by untreated cells, whereas the treatment with dexamethasone led to a reduction by 0.7 -fold $(\mathrm{p}=0.002)$ in the secreted CCL12 protein (Figure $2 \mathrm{~B}$ ). These results agree with those from the mRNA analysis in cEND cells after dexamethasone treatment as shown in Figure 1.

Confluent and differentiated cEND cells were treated for 24 hours with $100 \mathrm{nM}$ dexamethasone (dex) in serum-free medium. Equal amounts of cell culture medium were collected, acetone-precipitated and loaded on $20 \%$ Tris-glycine gel, followed by Western blot using anti-Csf3 (A) or anti-CCL12 (B) antibody. As a loading control, a Coomassie stained gel has been used. A representative Western blot is shown. Optical density values of protein bands were calculated with the software Alpha View and data expressed as fold over untreated control, which was set as 1 . The data (mean $\pm \mathrm{SD}$ ) present the results of three independent experiments. Statistical analysis was performed by t-test; ${ }^{*} \mathrm{p}<0.05$.

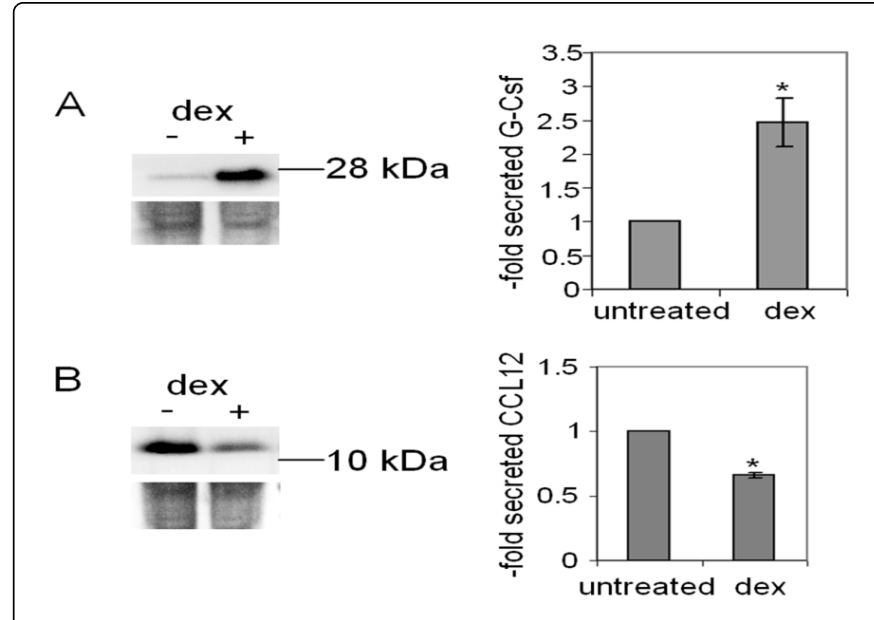

Figure 2: Dexamethasone-treated cEND release different levels of $\mathrm{G}-\mathrm{Csf}$ and CCL12 into the cell culture medium

\section{Pre-treatment of cEND with MS-patient sera leads to the higher dexamethasone-mediated G-Csf release from cEND treated with exacerbation phase serum}

As previously published by us, MS patient sera modify dexamethasone-mediated effects on blood-brain barrier properties [16]. We thus tested, whether pre-treatment of cEND with MS patient sera from different stages of the disease could influence the secretion of G-Csf and CCL12 into the culture medium (Figure 3A and 3B).

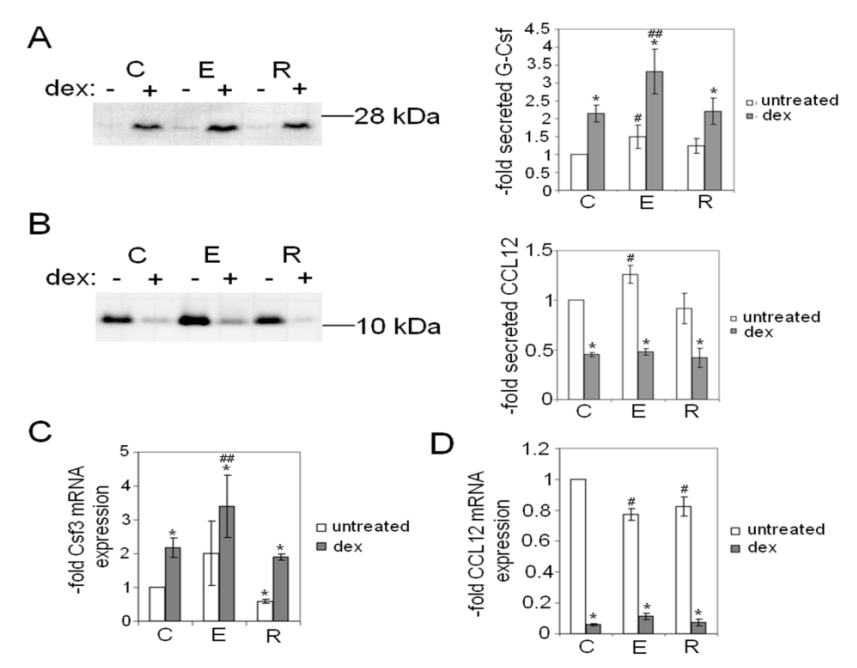

Figure 3: Secretion of G-CSF and CCL12 into the culture medium by cEND pre-treated with MS patient serum with or without dexamethasone treatment

Pre-treatment of cEND with control serum or MS patient serum from exacerbation phase revealed a 1.5 -fold $(\mathrm{p}<0.05)$ higher release of G-Csf in samples treated with MS patient serum from exacerbation phase, as detected by Western blot (Figure 3A). Interestingly, dexamethasone induction of G-Csf release was also 1.5 -fold $(\mathrm{p}<0.05)$ higher in exacerbation phase sera pre-treated cells than in control 
serum pre-treated samples (Figure 3A). CCL12 release into the medium was increased in samples treated with serum from exacerbation phase by 1.3 -fold $(\mathrm{p}<0.05)$ if compared to the cells treated with control serum (Figure $3 \mathrm{~B}$ ). The changes in G-Csf on the protein level correlated with Csf3 mRNA expression in cEND cells (Figure 3C). Csf3 mRNA expression was 1.6-fold $(\mathrm{p}<0.05)$ higher in dexamethasone samples pre-treated with exacerbation serum than in dexamethasone samples pre-treated with the control serum. However, CCL12 mRNA expression (Figure 3D) was decreased in samples pretreated with both exacerbation $(0.77$-fold, $\mathrm{p}<0.05)$ and remission $(0.82$-fold, $\mathrm{p}<0.05)$ phase patient sera if compared to sample pretreated with the control patient serum.

cEND cells were treated with MS patient serum (E- exacerbation phase, R- remission phase) or healthy control serum (C) for 24 hours, followed by PBS washing and addition of serum-free medium with or without $100 \mathrm{nM}$ dexamethasone (dex) for additional 24 hours. Equal amounts of the cell culture medium were collected, the proteins were precipitated with acetone and loaded on $20 \%$ Tris-glycine gel for electrophoresis followed by Western blot with anti-Csf3 (A) and antiCCL12 (B) antibody. Densitometric analyses of Western blots are shown at right. The data (mean $\pm \mathrm{SD}$ ) present the results of three independent experiments. Representative Western blots are shown. Statystical analysis was performed by 2 -way ANOVA followed by Tukey post hoc test; ${ }^{*} \mathrm{p}<0.05$ for comparisons untreated vs. dexamethasone-treated samples within different serum groups, \# $\mathrm{p}<0.05$ for comparisons of dexamethasone-untreated control and patient sera samples, \#\# $\mathrm{p}<0.05$ for comparisons of dexamethasonetreated control and patient sera samples. (C and D) Messenger RNA expression of Csf3 (C) and CCL12 (D) by cEND pre-treated with MS patient serum with or without dexamethasone treatment. cEND cells were treated with MS patient serum (E- exacerbation phase, Rremission phase) or healthy control serum (C) for 24 hours, followed by PBS washing and addition of serum-free medium with or without $100 \mathrm{nM}$ dexamethasone (dex) for additional 24 hours.

Csf3 and CCL12 expressions were normalized to GAPDH and expressed as fold over with control-serum pre-treated and dexamethasone untreated cells, which was set as 1 . The data (mean \pm SD) present the results of three independent experiments. Statystical analysis was performed by 2-way ANOVA followed by Tukey post hoc test; ${ }^{*} \mathrm{p}<0.05$ for comparisons untreated vs. dexamethasone-treated samples within different serum groups, \# $\mathrm{p}<0.05$ for comparisons of dexamethasone-untreated control and patient sera samples, \#\# $\mathrm{p}<0.05$ for comparisons of dexamethasone-treated control and patient sera samples.

\section{Mouse brain microvascular endothelial cells express chemokine (C-C motif) receptor 2 and Csf 3 receptor mRNA}

After showing the dexamethasone-mediated effects on Csf3 and CCL12, we examined whether cEND cells express the receptors for GCsf and CCL12 (Figure 4). G-Csf binds Csf3R, [24], and CCL12 interact with chemokine (C-C motif) receptor 2 (Ccr2), which also binds CCL2, CCL7, CCL8 and CCL16 [25]. cEND cells express high levels of mRNA for both receptors at a basal level. Analyses of Csf3R mRNA revealed strong dexamethasone-mediated down-regulation by 0.2 -fold $(\mathrm{p}<0.01)$, as compared to control untreated cells set as 1 (Figure 4A). No significant changes in Ccr2 mRNA expression were observed in cEND after dexamethasone treatment (Figure 4A). However, both receptors were up-regulated at the protein level in dexamethasone treated cEND (Csf3R by 2 -fold and Ccr2 by 1.7 -fold, respectively) as shown by Western blot in Figure 4B.

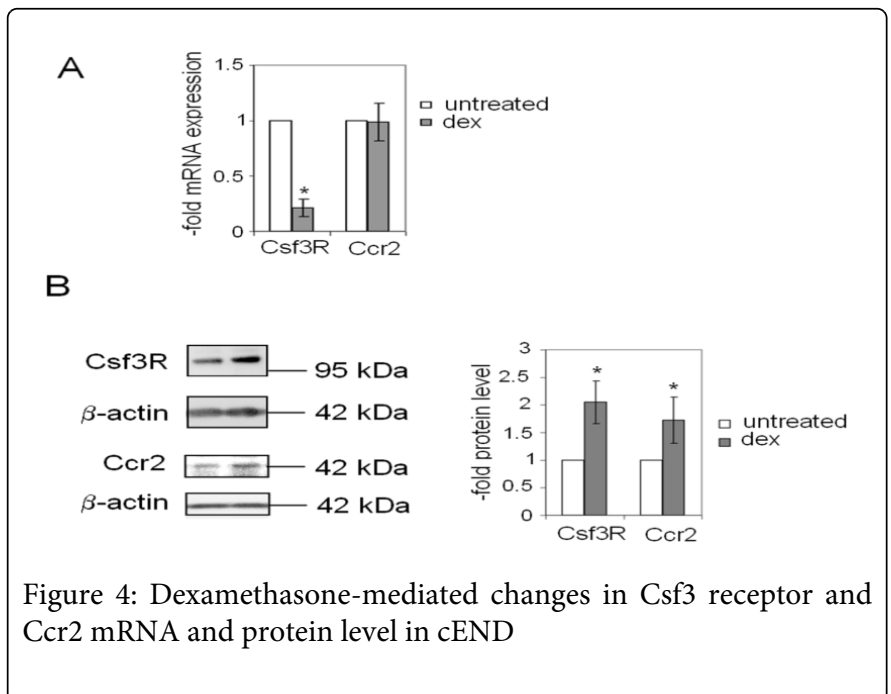

A. cEND cells were treated with $100 \mathrm{nM}$ dexamethasone for 24 hours followed by total RNA extraction and quantitative real-time PCR with gene specific primers. Csf3 receptor (Csf3R) and chemokine (C-C motif) receptor 2 (Ccr2) expression was normalized to GAPDH and expressed as fold over untreated cells, which was set as 1. B. Cell lysates form cEND treated or untreated with $100 \mathrm{nM}$ dexamethasone (dex) were subjected to SDS-PAGE as described in Materials and Methods. Expression of Csf3R and Ccr2 were detected with specific antibodies. A representative Western blot is shown. Optical density values of protein bands were calculated with the software Alpha View and data expressed as fold over untreated control, which was set as 1 . The data (mean $\pm \mathrm{SD}$ ) present the results of three independent experiments. Statistical analysis was performed by t-test; ${ }^{\star} \mathrm{p}<0.05$.

\section{Discussion}

Blood-brain barrier stabilizing effects of GCs have been previously reported $[10,16,26]$. However, the molecular mechanisms of these effects, especially in different neuroinflammatory disorders, are not completely understood. Cytokines are important mediators which contribute to the immune cell trafficking and functional integrity of different tissues. To further elucidate cytokine regulation in brain microvascular endothelial cells, we designed experiments aiming at providing an expression profile of cytokines in endothelial cells and, subsequently, identifying possible GC targets among cytokines expressed in brain endothelial cells in vitro. In our analysis, we included an in vitro blood-brain barrier model, the immortalized endothelial cells from mouse brain, cEND. This cell line has been previously proven to possess typical endothelial properties $[10,20]$. GC effects on endothelial barrier functions and the expression of glucocorticoid receptor (GR) have been also previously demonstrated $[10,16]$. In the present study, we characterized the cytokine mRNA expression profile of cEND cells at a basal level and after dexamethasone treatment. Moreover, we showed the dexamethasoneand MS patient sera mediated regulation of G-Csf and CCL12 in cEND.

First, we analyzed the mRNA products for different cytokines genes constitutively expressed in differentiated cEND without treatment with dexamethasone. Expression of tumor necrosis factor- $\alpha$ (Tnf- $\alpha$ ), 
Page 6 of 8

interleukin $1 \alpha$ and (Illa, Illb) and its receptor Ilr1, have been previously described in mouse brain microvascular ECs $[27,28]$. Also the expression of interleukins known to play a role in the pathogenesis of MS could be detected, e.g. Il6, Il12, Il17, Il25, as well as the receptors Il6ra and Il17ra [29]. Constitutive expression of CCL2 (MCP-1) [30] has been described in brain microvascular ECs and consistently, CCL2 mRNA was detected by us in cEND. The chemokines of CXC and CC subfamilies, which have been described in MS, including CCL2, -3, -4, $-5,-7$, and CXCL10, were also constitutively expressed by CEND [31]. The detection of gene expression for multiple cytokines and their receptors in cEND make this in vitro model of $\mathrm{BBB}$ a valuable tool for studying the leukocyte-endothelial adhesion and motility, as well as $\mathrm{BBB}$ properties under inflammatory conditions which are one of the characteristics of MS.

Glucocorticoids are the mainstay in the treatment of MS relapses [1]. We therefore tested dexamethasone-mediated changes in cytokine expression of cEND using qPCR-based arrays. We identified upregulation of mRNA expression in Csf3, Il17f, CXCL22, CXCL12, Il2rb and Il23a and a down-regulation of mRNA expression in CCL12, CXCR3, Kit and CCL7 after dexamethasone treatment. CXCL2 (MIP-2, macrophage inflammatory protein-2) has been up-regulated by dexamethasone in traumatic brain injury in cells with neuronal morphology [32]. Our results demonstrated that CXCL2 mRNA was highly induced by dexamethasone in brain microvascular EC. CXCL12 (Sdf-1, stromal cell-derived factor-1) was up-regulated by dexamethasone in our system (Table 1 and Figure 2). CXCL12 has been demonstrated to be expressed in healthy adult CNS on neurons and endothelial cells and it was up-regulated in MS lesions [33] suggesting its important role in MS pathology. GC therapy of MS patients reduced CXCR3 (a known receptor for CXCL9, CXCL10 and CXCL11) expression on peripheral blood T cells, thereby diminishing the recruitment of leukocytes into the CNS [34]. We also showed GCmediated down-regulation of CXCR3 on brain microvascular ECs, which might contribute to beneficial effects of GC in MS. To authors' best knowledge, this is the first report showing dexamethasonemediated regulation of CCL12 and Il23a. Il23 stimulates the production of interferon gamma and Il10 having an anti-inflammatory function $[35,36]$.

The most prominent changes in the expression were detected for Csf3 and CCL12 mRNA and protein after dexamethasone treatment. CCL12 (Mcp-5) is a proinflammatory chemokine, which was as expected down-regulated by dexamethasone, as already shown for other chemokines from the CC subfamily [30]. CCL12 protein and mRNA expression has been previously demonstrated in mouse brain and mouse endothelial cell of aorta [23]. cEND cells express and secrete high levels of CCL12 constitutively. Pre-treatment of cEND with MS-patient sera changed the levels of CCL12 mRNA and protein in cEND, suggesting an ability of cEND to answer to different components present in MS-patient serum in comparison to serum from healthy donor. CCL12, together with CCL2 have been shown to play a role in the induction of inflammatory response in cyrolesionEAE (experimental autoimmune encephalomyelitis) as their expression was the highest before the maximum degree of inflammation in the brain was seen [37]. Moreover, multiple sequence polymorphisms resulting in significant amino acid substitutions were identified in CCL12 gene, which was associated with severity of clinical signs and susceptibility to less severe monophasic remitting/ nonrelapsing form of EAE [38]. Here, we identified CCL12 as a target gene for GC-action in brain vascular endothelium. Dexamethasone preserves its strong inhibiting effects on CCL12 mRNA and protein in the cells pre-treated with MS patient sera, which might be of interest for MS therapy. Expression of CCL12 receptor, Ccr2 was detected in cEND at the protein and mRNA level. Mice lacking Ccr2 receptors were resistant to the induction of EAE by myelin oligodendrocyte glycoprotein peptide 35-55 [39].

G-Csf is a key hemopoietic factor of the myeloid lineage and has been clinically used in the last decade for treatment of neutropenia and mobilization of bone marrow normal hemopoietic stem cells into the peripheral blood [22]. In microarray study with MS patient samples, G-Csf mRNA was identified as upregulated in acute, but not in chronic MS lesions and treatment of EAE mice with G-Csf ameliorated the disease [40] or reversed ongoing EAE [41]. Here, we demonstrate that mouse brain microvascular endothelial cells secrete high amounts of G-Csf into medium in vitro after stimulation with dexamethasone. Basal G-Csf secretion takes place, although it is hardly detectable. G-Csf is also constitutively secreted by mouse microvascular brain pericytes, which together with endothelial cells and astrocytes are a part of the neurovascular unit [42]. The treatment of cEND cells with MS patient serum from exacerbation phase led to a higher basal G-Csf secretion which was potentiated after treatment with dexamethasone. We demonstrated that cEND cells expressed GCsf receptor on mRNA and protein level indicating that brain vascular endothelium may play an important role in naturally suppressing acute attacks of MS.

\section{Conclusions}

In conclusion, our results as presented here show that cultured mouse brain endothelial cells express high levels of cytokines and chemokines mRNAs and respond to dexamethasone stimulation. Mouse brain endothelial cells express and secrete in vitro CCL12 und Csf3 (G-CSF) and their receptors, thus being able to transduce signals triggered during brain inflammatory or neurodegenerative processes. Additionally, we showed that sera from multiple sclerosis patients influence the mRNA expression and secretion of CCL12 and G-CSF. CCL12 and Csf3 can be assumed as novel dexamethasone targets in brain vascular endothelium.

\section{Acknowledgments}

This research was partly supported by the European Union Seventh Framework Programme (FP7/2007-2013) grant under the grant agreement HEALTH-F2-2009-241778 to CYF and MB. The authors are grateful to Elisabeth Wilken for excellent technical assistance.

\section{Authors' contributions}

MB designed the study, acquired, analyzed and interpreted the data and wrote the manuscript. AH, RG, AC collected and provided the patient sera and critically revised the manuscript. NR and CYF interpreted the data and critically revised the manuscript. All authors read and approved the final manuscript.

\section{References}

1. Tischner D, Reichardt HM (2007) Glucocorticoids in the control of neuroinflammation. Mol Cell Endocrinol 275: 62-70.

2. Steinman L (2001) Multiple sclerosis: a two-stage disease. Nat Immunol 2: $762-764$.

3. Engelhardt B (2006) Molecular mechanisms involved in T cell migration across the blood-brain barrier. J Neural Transm 113: 477-485. 
4. SzczuciÅ, ski A, Losy J (2007) Chemokines and chemokine receptors in multiple sclerosis. Potential targets for new therapies. Acta Neurol Scand 115: 137-146.

5. Mun-Bryce S, Rosenberg GA (1998) Matrix metalloproteinases in cerebrovascular disease. J Cereb Blood Flow Metab 18: 1163-1172.

6. Benesová Y, Vasku A, Novotná H, Litzman J, Stourac P, et al. (2009) Matrix metalloproteinase-9 and matrix metalloproteinase-2 as biomarkers of various courses in multiple sclerosis. Mult Scler 15: 316-322.

7. Reichardt HM (2004) Immunomodulatory activities of glucocorticoids: insights from transgenesis and gene targeting. Curr Pharm Des 10: 2797-2805.

8. Wüst S, van den Brandt J, Tischner D, Kleiman A, Tuckermann JP, et al. (2008) Peripheral T cells are the therapeutic targets of glucocorticoids in experimental autoimmune encephalomyelitis. J Immunol 180: 8434-8443.

9. Sbiera S, Dexneit T, Reichardt SD, Michel KD, van den Brandt J, et al. (2011) Influence of short-term glucocorticoid therapy on regulatory $\mathrm{T}$ cells in vivo. PLoS One 6: e24345.

10. Forster C, Silwedel C, Golenhofen N, Burek M, Kietz S, et al. (2005) Occludin as direct target for glucocorticoid-induced improvement of blood-brain barrier properties in a murine in vitro system. J Physiol 565: 475-486.

11. Rosenberg GA, Dencoff JE, Correa N Jr, Reiners M, Ford CC (1996) Effect of steroids on CSF matrix metalloproteinases in multiple sclerosis: relation to blood-brain barrier injury. Neurology 46: 1626-1632.

12. Lindberg RL, De Groot CJ, Montagne L, Freitag P, van der Valk P, et al. (2001) The expression profile of matrix metalloproteinases (MMPs) and their inhibitors (TIMPs) in lesions and normal appearing white matter of multiple sclerosis. Brain 124: 1743-1753.

13. Murphy PM, Baggiolini M, Charo IF, Hebert CA, Horuk R, et al. (2000) International union of pharmacology. XXII. Nomenclature for chemokine receptors. Pharmacol Rev 52: 145-176.

14. Subileau EA, Rezaie P, Davies HA, Colyer FM, Greenwood J, et al. (2009) Expression of chemokines and their receptors by human brain endothelium: implications for multiple sclerosis. J Neuropathol Exp Neurol 68: 227-240.

15. Förster C, Waschke J, Burek M, Leers J, Drenckhahn D (2006) Glucocorticoid effects on mouse microvascular endothelial barrier permeability are brain specific. J Physiol 573: 413-425.

16. Blecharz KG, Haghikia A, Stasiolek M, Kruse N, Drenckhahn D, et al. (2010) Glucocorticoid effects on endothelial barrier function in the murine brain endothelial cell line cEND incubated with sera from patients with multiple sclerosis. Mult Scler 16: 293-302.

17. Burek M, Salvador E, Förster CY (2012) Generation of an immortalized murine brain microvascular endothelial cell line as an in vitro blood brain barrier model. J Vis Exp : e4022.

18. Berger O, Gan X, Gujuluva C, Burns AR, Sulur G, et al. (1999) CXC and $\mathrm{CC}$ chemokine receptors on coronary and brain endothelia. Mol Med 5 : 795-805.

19. Hillyer P, Mordelet E, Flynn G, Male D (2003) Chemokines, chemokine receptors and adhesion molecules on different human endothelia: discriminating the tissue-specific functions that affect leucocyte migration. Clin Exp Immunol 134: 431-441.

20. Silwedel C, Forster C (2006) Differential susceptibility of cerebral and cerebellar murine brain microvascular endothelial cells to loss of barrier properties in response to inflammatory stimuli. J Neuroimmunol 179: 37-45.

21. Burek M, Förster CY (2009) Cloning and characterization of the murine claudin-5 promoter. Mol Cell Endocrinol 298: 19-24.

22. Rutella S, Zavala F, Danese S, Kared H, Leone G (2005) Granulocyte colony-stimulating factor: a novel mediator of $\mathrm{T}$ cell tolerance. J Immunol 175: 7085-7091.

23. Rodríguez-Sanabria F, Rull A, Beltrán-Debón R, Aragonès G, Camps J, et al. (2010) Tissue distribution and expression of paraoxonases and chemokines in mouse: the ubiquitous and joint localisation suggest a systemic and coordinated role. J Mol Histol 41: 379-386.

24. Germeshausen M, Skokowa J, Ballmaier M, Zeidler C, Welte K (2008) GCSF receptor mutations in patients with congenital neutropenia. Curr Opin Hematol 15: 332-337.

25. Semple BD, Kossmann T, Morganti-Kossmann MC (2010) Role of chemokines in CNS health and pathology: a focus on the CCL2/CCR2 and CXCL8/CXCR2 networks. J Cereb Blood Flow Metab 30: 459-473.

26. Förster C, Burek M, Romero IA, Weksler B, Couraud PO, et al. (2008) Differential effects of hydrocortisone and TNFalpha on tight junction proteins in an in vitro model of the human blood-brain barrier. J Physiol 586: 1937-1949.

27. Banks WA (1999) Characterization of interleukin-1alpha binding to mouse brain endothelial cells. J Pharmacol Exp Ther 291: 665-670.

28. Zhang W, Smith C, Howlett C, Stanimirovic D (2000) Inflammatory activation of human brain endothelial cells by hypoxic astrocytes in vitro is mediated by IL-1beta. J Cereb Blood Flow Metab 20: 967-978.

29. Codarri L, Fontana A, Becher B (2010) Cytokine networks in multiple sclerosis: lost in translation. Curr Opin Neurol 23: 205-211.

30. Harkness KA, Sussman JD, Davies-Jones GA, Greenwood J, Woodroofe MN (2003) Cytokine regulation of MCP-1 expression in brain and retinal microvascular endothelial cells. J Neuroimmunol 142: 1-9.

31. Trebst C, Ransohoff RM (2001) Investigating chemokines and chemokine receptors in patients with multiple sclerosis: opportunities and challenges. Arch Neurol 58: 1975-1980.

32. Rhodes JK, Sharkey J, Andrews PJ (2009) The temporal expression, cellular localization, and inhibition of the chemokines MIP-2 and MCP-1 after traumatic brain injury in the rat. J Neurotrauma 26: 507-525.

33. Krumbholz M, Theil D, Cepok S, Hemmer B, Kivisäkk P, et al. (2006) Chemokines in multiple sclerosis: CXCL12 and CXCL13 up-regulation is differentially linked to CNS immune cell recruitment. Brain 129: 200-211.

34. Sørensen TL, Trebst C, Kivisäkk P, Klaege KL, Majmudar A, et al. (2002) Multiple sclerosis: a study of CXCL10 and CXCR3 co-localization in the inflamed central nervous system. J Neuroimmunol 127: 59-68.

35. Oppmann B, Lesley R, Blom B, Timans JC, Xu Y, et al. (2000) Novel p19 protein engages IL-12p40 to form a cytokine, IL-23, with biological activities similar as well as distinct from IL-12. Immunity 13: 715-725.

36. de Paus RA, van de Wetering D, van Dissel JT, van de Vosse E (2008) IL-23 and IL-12 responses in activated human $\mathrm{T}$ cells retrovirally transduced with IL-23 receptor variants. Mol Immunol 45: 3889-3895.

37. Sun D, Tani M, Newman TA, Krivacic K, Phillips M, et al. (2000) Role of chemokines, neuronal projections, and the blood-brain barrier in the enhancement of cerebral EAE following focal brain damage. J Neuropathol Exp Neurol 59: 1031-1043.

38. Teuscher C, Butterfield RJ, Ma RZ, Zachary JF, Doerge RW, et al. (1999) Sequence polymorphisms in the chemokines Scyal (TCA-3), Scya2 (monocyte chemoattractant protein (MCP)-1), and Scya12 (MCP-5) are candidates for eae7, a locus controlling susceptibility to monophasic remitting/nonrelapsing experimental allergic encephalomyelitis. J Immunol 163: 2262-2266.

39. Izikson L, Klein RS, Charo IF, Weiner HL, Luster AD (2000) Resistance to experimental autoimmune encephalomyelitis in mice lacking the CC chemokine receptor (CCR)2. J Exp Med 192: 1075-1080.

40. Lock C, Hermans G, Pedotti R, Brendolan A, Schadt E, et al. (2002) Gene-microarray analysis of multiple sclerosis lesions yields new targets validated in autoimmune encephalomyelitis. Nat Med 8: 500-508.

41. Zavala F, Abad S, Ezine S, Taupin V, Masson A, et al. (2002) G-CSF therapy of ongoing experimental allergic encephalomyelitis via chemokine- and cytokine-based immune deviation. J Immunol 168: 2011-2019.

42. Kovac A, Erickson MA, Banks WA (2011) Brain microvascular pericytes are immunoactive in culture: cytokine, chemokine, nitric oxide, and LRP-1 expression in response to lipopolysaccharide. J Neuroinflammation 8: 139. 